\title{
Radial osteomyelitis as a complication of venous cannulation
}

\author{
R Straussberg, L Harel, Z Bar-Sever, J Amir
}

\begin{abstract}
Venepuncture of the superficial veins in the forearm is considered a relatively safe procedure. We report two patients who presented with osteomyelitis of the proximal radius following venous cannulation of the median cubital vein, and one patient who developed osteomyelitis of the distal radius after cannulation of the cephalic vein. Osteomyelitis developing in proximity to a venepuncture site should raise the suspicion that a pathogen causing superficial thrombophlebitis has spread through the deep veins of the arm into the adjacent bone, thus causing osteomyelitis. (Arch Dis Child 2001;85:408-410)
\end{abstract}

Keywords: osteomyelitis; venous cannulation; thrombophlebitis

Venous cannulation of the superficial forearm veins is a common procedure. The visible and accessible median cubital vein is a preferred site for insertion of an intravenous line. In the majority of cases, the procedure is without complications, although superficial thrombophlebitis is sometimes a sequela. Rarely, infection of the median cubital vein spreads to the deep venous system of the arm.

We report a rare complication of catheterisation of the superficial veins of the forearm (the median cubital and cephalic veins). This caused proximal and distal radial osteomyelitis, respectively. The infection developed at the site of venepuncture, suggesting that it spread locally through anastomoses between the superficial medial cubital and cephalic veins and the deep radial vein. Whenever point tenderness develops over a long bone, over the underlying skin, after venepuncture, osteomyelitis should be suspected.

Schneider Children's

Medical Center of

Israel, Petah Tikva,

Israel 49202

R Straussberg

L Harel

J Amir

Department of

Nuclear Imaging,

Schneider Children's

Medical Center of

Israel, Petah Tikva and

Sackler School of

Medicine, Tel Aviv

University, Tel Aviv,

Israel

Z Bar-Sever

Correspondence to:

Dr Straussberg

rachelst@post.tau.ac.il

Accepted 20 July 2001 and ankle joints. He was treated regularly with colchicine $1.5 \mathrm{mg} /$ day. Seven days prior to admission to our hospital, he was hospitalised elsewhere with pneumonia. Treatment consisted of cefuroxime administered through a "Quickcath" inserted in the left median cubital vein. Blood culture was negative. $\mathrm{He}$ was discharged after four days and prescribed oral roxithromycin $150 \mathrm{mg}$ twice daily.

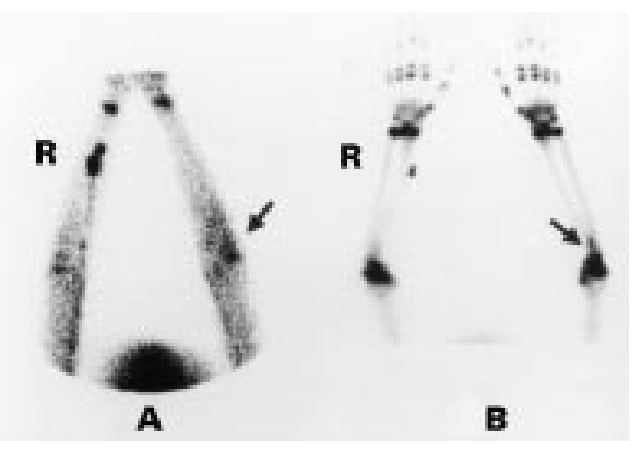

Figure 1 Tissue phase image (A) from a three phase bone scan shows diffuse, increased tracer localisation in the proximal left forearm (arrow), suggesting soft tissue hyperaemia adjacent to the elbow. The skeletal phase image (B) shows abnormal focal uptake in the proximal left radius (arrow). These findings are consistent with osteomyelitis. The focal uptake seen in the radial aspect of the right forearm on both images is an injection site artefact.

On the day following the venepuncture performed at the other hospital, the patient began to complain of pain in the elbow which was different from the pain he had felt during previous episodes of arthritis. On the day of admission to our hospital, the pain had become excruciating.

On physical examination, he was afebrile. The left elbow was swollen. Redness and tenderness were noted over the left median cubital vein, compatible with superficial thrombophlebitis. There was point tenderness over the proximal aspect of the left radius and incomplete range of motion was elicited in the left elbow. There was no extravasation around the cannula. The white cell count was $1.5 \times 10^{9}$ cells $/ \mathrm{mm}^{3}$ with a differential count of $74 \%$ polymorphonucleocytes, $22 \%$ lymphocytes, $3 \%$ monocytes, and $1 \%$ eosinophils. Sedimentation rate was $51 \mathrm{~mm} / \mathrm{h}$ (Westergren), serum $\mathrm{C}$ reactive protein (CRP) was $7.4 \mu \mathrm{g} / 1$ (normal $<0.5 \mu \mathrm{g} / \mathrm{l})$. Blood culture was sterile. Nuclear imaging was compatible with a diagnosis of osteomyelitis of the left proximal radius near the elbow joint (fig 1). The patient was treated with intravenous cefazolin $1.5 \mathrm{~g}$, three times daily for 10 days, with complete resolution of symptoms. CRP decreased to $1.9 \mu \mathrm{g} / \mathrm{l}$. He was discharged and prescribed oral cephalexin 2.5 $\mathrm{g} /$ day for two weeks.
CASE 2

A 3 year old girl was admitted with fever of $39.2^{\circ} \mathrm{C}$. Urine culture yielded Escherichia coli and a diagnosis of urinary tract infection was made. After cleaning the skin overlying the inner aspect of the elbow with a solution of $70 \%$ alcohol, an intravenous catheter was inserted into the right median cubital vein for 
administration of gentamicin. The next day body temperature returned to normal. The intravenous line was transferred to the contralateral side after three days. However, after five days of treatment, the temperature rose again, and the parents noted the refusal of the child to move her right arm.

On physical examination the skin overlying the right elbow was warmer than the contralateral side, and there was point tenderness over the medial aspect of the proximal radius. There was no extravasation around the cannula. White blood cell count was $1.7 \times 10^{9}$ cells/ $\mathrm{mm}^{3}$ with a differential count of $68 \%$ polymorphonucleocytes, $20 \%$ lymphocytes, 3\% eosinophils, $8 \%$ monocytes, and $1 \%$ basophils. An $x$ ray examination of the arm was interpreted as normal. Sedimentation rate was $72 \mathrm{~mm} / \mathrm{h}$ (Westergren). Blood culture was sterile. Radionuclide imaging showed pathological uptake of the isotope in the right proximal radius at the late phase of the examination. A diagnosis of osteomyelitis was made. The patient was treated with cloxacillin $1.5 \mathrm{~g}$ per day intravenously for three weeks with complete resolution of symptoms. At discharge she was prescribed oral cephalexin $1.5 \mathrm{~g} /$ day for an additional two weeks.

CASE 3

An 18 year old female with insulin dependent diabetes mellitus (diagnosed at the age of 8 years and treated with subcutaneous insulin), was admitted to our hospital with fever, sore throat, and abdominal pain; there was laboratory evidence of ketoacidosis and pharyngitis was diagnosed. After cleaning the skin over the distal end of the forearm with a solution of $70 \%$ alcohol, an intravenous line was inserted into the cephalic vein; treatment with intravenous fluids and insulin was initiated. Initial leucocyte count was $0.8 \times 10^{9}$ cells $/ \mathrm{mm}^{3}$ with $63 \%$ polymorphonucleocytes, $20 \%$ lymphocytes, $5 \%$ monocytes, $10 \%$ eosinophils, and $2 \%$ basophils. The pharyngitis was believed to be viral. On the third day her temperature returned to normal; she was treated with subcutaneous insulin, but continued to be hospitalised because of unstable blood glucose concentrations.

On the fifth day her temperature rose to $39.6^{\circ} \mathrm{C}$ and she complained of pain in the distal aspect of the radius. On examination there were signs of phlebitis - the skin over the intravenous insertion was warm and red but point tenderness was elicited only secondary to strong pressure on the area of the styloid process of the radius. There was no extravasion around the cannula. Blood count was $1.3 \times 10^{9}$ leucocytes per $\mathrm{mm}^{3}$ with a differential count of $63 \%$ polymorphonucleocytes, $17 \%$ lymphocytes, $11 \%$ monocytes, $6 \%$ eosinophils, and $3 \%$ basophils. Sedimentation rate was 94 $\mathrm{mm} / \mathrm{h}$ (Westergren); serum CRP was 11.1 $\mu \mathrm{g} / \mathrm{dl}$. Blood culture was negative. The intravenous line was transferred to another site. A technetium bone scan in the bony phase suggested increased uptake of the colloid in the styloid process of the distal radius, supporting a diagnosis of osteomyelitis (fig 2).

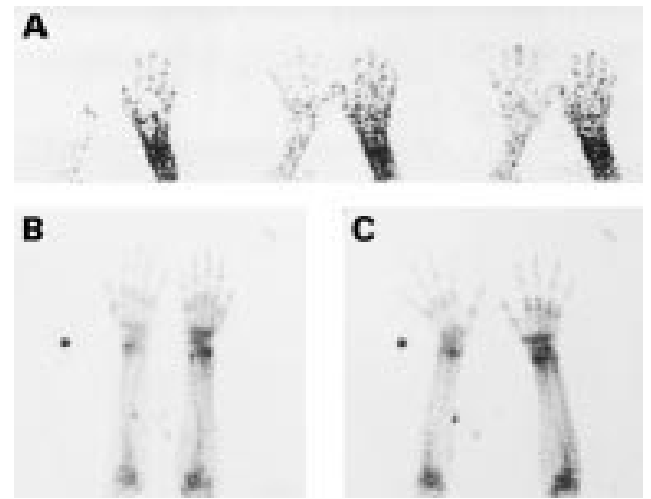

Figure 2 Dynamic images $(A)$ from the angiographic phase of a bone scan show increased blood flow to the left wrist. Dorsal (B) and palmar (C) views of the hands from the skeletal phase of the study show diffuse, increased uptake in the region of the left wrist and carpus, consistent with cellulitis. There is some prominence in the appearance of the styloid process of the radius, suggesting osteomyelitis.

Treatment with vancomycin $50 \mathrm{mg} / \mathrm{kg} /$ day in three divided doses and garamicin $5 \mathrm{mg} / \mathrm{kg} /$ day as a single dose, was commenced. Temperature returned to normal after three days and the patient ceased to complain of pain after two days.

\section{Discussion}

The veins of the upper arm are frequently used for drawing blood for intravenous injections and infusions. The median cubital vein is commonly used for venepuncture and for cannulation because it is easily accessible and allows communication between the basilic and cephalic veins, through which superficial venous drainage of the forearm occurs. The basilic vein penetrates the deep fascia on the medial side of the middle part of the arm and then joins the brachial veins to form the axillary vein. Numerous deep veins drain the structures of the forearm. They arise from a deep venous arcade (a series of anastomosing venous arches) in the hand. The deep veins ascend the forearm along the sides of the corresponding arteries, receiving tributaries from veins leaving the related muscles and communicating with superficial veins. The deep interosseous veins that accompany the respective arteries unite with the accompanying veins of the radial and ulnar arteries. The deep veins in the cubital fossa are connected to the median cubital vein and unite with the accompanying veins of the respective artery. ${ }^{1}$

In the first two patients, osteomyelitis of the proximal radius developed after venepuncture and insertion of an intravenous line through the median cubital vein. In the third patient, osteomyelitis of the distal radius developed as a consequence of cannulating the cephalic vein. Although the bacterial aetiology was not defined, we believe that the diagnosis of osteomyelitis was established based on the clinical findings and the results of the nuclear imaging. We were able to find several previous reports of osteomyelitis as a complication of venepuncture. $^{2-6}$ The site of osteomyelitis was the clavicle in all cases, and the infection developed following subclavian vein catheterisation. The 
investigators assumed that the causative organisms were inoculated directly into the clavicular periosteum and did not propagate from distant foci. ${ }^{5}$ We have found two additional reports, of osteomyelitis secondary to multiple punctures of the great toe for draining blood in a premature infant, ${ }^{7}$ and following needle puncture in two neonates. ${ }^{8}$ We suggest that in our three cases, bacteria, possibly Staphylococcus aureus from the overlying skin inoculated during the venepuncture, caused superficial thrombophlebitis of the median cubital vein and the cephalic vein. From there, the infection spread to the deep venous system of the forearm, via the radial veins through their anastomoses with the median cubital vein and the cephalic vein, and then to the adjacent bone itself. The fact that osteomyelitis developed in the site of the venepuncture and not in a distant locus suggests that there was a local infection and not bacteraemia with distant dissemination. The blood cultures in our three patients were negative, thus ruling out a pneumonia causing agent or $E$ coli bacteraemia as aetiologies for the osteomyelitis. There were no signs of superinfection around the cannula site which extended down into deep tissues with direct continuous spread. The inflamed area appeared to be thrombophlebitis and not local cellulitis.

We believe that this complication of intravenous line insertion has not been reported previously in the English literature. We conclude that osteomyelitis should be suspected whenever point tenderness over a long bone develops after venepuncture.

1 Moore KI, Dalley AF. Forearm. In: Kelly PJ, ed. Clinically oriented anatomy, 4 th edn. Baltimore: Lippincott Williams \& Wilkins, 1999:734-57.

2 Lee Y-H, Kerstein MD. Osteomyelitis and septic arthritis: a complication of subclavian venous catheterization. N Engl f Med 1971;285:79-80.

3 Manny J, Haruzi I, Yosipovitch Z. Osteomyelitis of the clavicle following subclavian vein catheterization. Arch Surg 1973;106:342-3.

4 Steein J, Pederson JHJ. Osteomyelitis of the clavicle following percutaneous subclavian catheterization. Dan Med Bull 1978;25:260-1.

5 Klein B, Mittelman M, Katz R, Djaldetti M. Osteomyelitis of both clavicles as a complication of subclavian venipuncture. Chest 1983;83:143-4.

6 Garcia S, Combalia A, Segur JM, Llovera AJ. Osteomyelitis of the clavicle. A case report. Acta Orthop Belg 1999;65: 369-71.

7 Puczynski MS, Dvonch VM, Menendez CE, Caldwell CC. Osteomyelitis of the great toe secondary to phlebotomy. Clin Orthop 1984:190:239-40.

8 Nelson DL, Hable KA, Matsen JM. Proteus mirabilis osteomyelitis in two neonates following needle puncture. Successful treatment with ampicilin. Am $\mathcal{F}$ Dis Child 1973; 125:109-10.

\section{Rapid responses}

Letters on the following papers have been published recently as rapid responses on the $A D C$ website. To read these letters visit www.archdischild.com and click on "Read eLetters"

Diagnostic assessment of haemorrhagic rash and fever. $\mathrm{H} \mathrm{E}$ Nielsen, $\mathrm{E}$ A Andersen, $\mathrm{J}$ Andersen, et al. Arch Dis Child 2001;85:160-5.

The child with a non-blanching rash: how likely is meningococcal disease? $\mathrm{L} C \mathrm{Wells}$, $\mathrm{C}$ Smith, V C Weston, et al. Arch Dis Child 2001;85:218-22.

Measurement of urinary constituents and output using disposable napkins. SB Roberts, A Lucas. Arch Dis Child 1985;60:1021-4.

Accidents and resulting injuries in premobile infants: data from the ALSPAC study. S A Warrington, C M Wright, ALSPAC Study Team. Arch Dis Child 2001;85:104-7.

If you would like to post an electronic response to these or any other articles published in the journal, please go to the website, access the article in which you are interested, and click on "eLetters: $\underline{\text { Submit a response to }}$ this article" in the box in the top right hand corner. 